\title{
Estudio Cualitativo en torno a la Aplicación del Modelo de Autoevaluación Asistida Basado en el uso de Plataformas Digitales, en un Curso de Pedagogía
}

\author{
Marcelo H. Rioseco y Andrew Philominraj \\ Universidad Católica del Maule, Av. San Miguel 3605, Talca -Maule (e-mail: mrioseco@ucm.cl; andrew@ucm.cl)
}

Recibido Ago. 24, 2018; Aceptado Nov. 20, 2018; Versión final Ene. 2, 2019, Publicado Jun. 2019

\begin{abstract}
Resumen
Se presenta una propuesta de evaluación educativa denominada modelo de autoevaluación asistida, basado en el uso de plataformas digitales. Así mismo, se describe la valoración que los estudiantes de un curso de pre-grado de una carrera de pedagogía hicieron de este modelo, al ser aplicado, durante un semestre, en la universidad. Esta valoración, fue abordada mediante un estudio cualitativo basado en entrevistas, aplicadas a 14 estudiantes, a través de un muestreo no probabilístico intencional. Las categorías que derivaron a partir del análisis, describen la valoración que los estudiantes hicieron de las actividades realizadas, la percepción que tuvieron de sus aprendizajes, del proceso de evaluación, del nivel de exigencia y la valoración que hicieron de la propuesta general del curso. Se concluye que es fundamental dotar de nuevo significado a la evaluación educativa, fomentando una mirada responsable del estudiante hacia su desempeño.
\end{abstract}

\section{Qualitative Study on the Application of the Assisted Self- Assessment Model Based on the use of Digital Platform in an Initial Teacher Formation Course}

\begin{abstract}
This article presents an educational evaluation proposal called assisted self-evaluation model based on the use of digital platforms. In addition, the article describes the assessment that the students of an undergraduate course of a pedagogy made of this model, when it was applied during a semester in a university. This assessment based on a qualitative study was carried out by interviews, applied to 14 students, through an intentional non-probabilistic sampling. The categories derived from the analysis describe the students' assessment of the activities carried out, their perception of their learning, the evaluation process, the level of demand and the assessment they made of the general proposal of the course. It is concluded that it is of paramount importance to give new meaning to educational evaluation, encouraging a responsible view of the students' performance.
\end{abstract}

Keywords: authentic assessment; digital platform; standardized tests; learning 


\section{INTRODUCCIÓN}

Según Barrenechea (2018), las evaluaciones estandarizadas han sido utilizadas para satisfacer diferentes necesidades en el campo educativo, aunque más recientemente este tipo de evaluaciones se han convertido en una pieza fundamental para delimitar la responsabilidad de las escuelas y de los educadores. En el caso chileno, se atribuye una importancia central a las pruebas estandarizadas, en la promoción de la calidad y de la equidad, aun cuando esta relación no está demostrada. Por el contrario, se ha visto que la segregación y la falta de calidad en el sistema chileno son problemas que tienden a profundizarse (Lipman, 2009).Cuando la evaluación estandarizada se utiliza para clasificar a los estudiantes, muchas veces se está desconociendo que el aprendizaje tiene lugar en un sujeto en situación, con una historia que determina aquello que es y que sabe, y con un futuro que da sentido y dirección a aquello que es y que sabe. La importancia que ha adquirido la estandarización en evaluación educativa, tiene sus raíces en las primeras utilizaciones de test estandarizados para cuestiones de análisis psicológico, en Estados Unidos, en la década de 1940 (Barrenechea, 2018). Uno de los atractivos que poseen estos test es que dan la idea de que es posible sistematizar nuestra manera de apreciar propiedades complejas, relacionadas con el comportamiento y tradicionalmente llamadas "subjetivas", a través de la cuantificación. Es necesario, sin embargo, considerar que la cuantificación de estas propiedades no permite, en sí misma, un tipo de descripción más objetiva. Lamentablemente, pareciera que en el ámbito de la educación continua prevaleciendo un punto de vista que da por hecho que las mediciones cuantitativas que se realizan poseen un carácter objetivo, en el sentido de "objetividad sin paréntesis" (Maturana, 2001).

El proceso de aprendizaje humano, en cambio, está lleno de matices, de saberes incompletos y de errores que permiten avanzar y perfeccionarse. En este sentido, en el aprendizaje significativo, el estudiante es considerado como persona que interpreta y da sentido a su ambiente y como un agente activo ante la información que recibe, generando experiencias, que reestructura y remodela lo que ya sabe para conseguir un nuevo aprendizaje (Quaas y Crespo, 2003). Se da prioridad, de esta manera, a las peculiaridades de cada individuo, y se toman en cuenta las diferencias de las personas ante el aprendizaje y las causas por las que unos realizan algo mejor que otros (Mojarro, Rodrigo, Etchegaray y Centeno 2015). Para la evaluación estandarizada, en cambio, el propósito es la clasificación y la comparación y las respuestas son correctas e incorrectas; las primeras se premian, las segundas se castigan. El error se penaliza; se emplea para clasificar y para estigmatizar, ya sea al estudiante, o al grupo de estudiantes: alumnos buenos o malos y grupos de alumnos, buenos o malos.

La evaluación estandarizada, si es aplicada de manera ingenua, resulta contraproducente para el aprendizaje, ya que, en primer lugar, tiende a desnaturalizar el saber, ya que puede fomentar que éste se desarrolle de manera abstracta, en contextos artificiales. Para llevar a cabo una evaluación estandarizada con grandes números de estudiantes es necesario, por lo general, aplicar pruebas escritas, que inducen a los alumnos a retener información y manejar contenidos, es decir, a incorporar un tipo de saber que esencialmente tiene sentido en el contexto de la prueba. En este sentido, Linn (1993) sostiene que, en muchas oportunidades, el incremento en los resultados en evaluaciones estandarizadas no tiene que ver con una profundización de los conocimientos, sino que se debe a una familiarización con la metodología de los exámenes. En segundo lugar, la evaluación estandarizada puede llegar a hacer equivalente el comportamiento humano al funcionamiento de una máquina. Una máquina puede ser descrita de acuerdo a su estructura y a su modo de operar, en un momento dado. Así mismo, es posible someter a la máquina a diferentes tipos de pruebas para constatar que está funcionando correctamente y que cumple con las expectativas que tenemos en relación a las tareas que realiza. Las mismas pruebas, pueden ser repetidas en varias máquinas, y los resultados nos permiten describirlas, compararlas y clasificarlas. El comportamiento humano, en cambio, no es reductible a fuerzas mecánicas, ya que tiene su origen en un sujeto que actúa, de manera intencional, en el mundo, de acuerdo a sus posibilidades y, entre ellas, a su saber (Álvarez, 2011). Este comportamiento no es un fenómeno que podamos objetivar del mismo modo como objetivamos las propiedades de una cosa, ya que se produce en contextos de interacción humana. La evaluación estandarizada, como se aplica en muchos sistemas educativos en la actualidad, mide y compara a los sujetos, con el objetivo principal de clasificarlos. Cuando esto ocurre, la educación formal, entonces, no tiene la función ni de educar, ni de formar, sino de "acreditar", de entregar credenciales para determinar diferentes tipos de acceso al mundo productivo.

\section{Aprendizaje a través de actividades}

Robbins y Judge (2009) define el aprendizaje como "cualquier cambio relativamente permanente en la conducta, como consecuencia de una experiencia". A través del aprendizaje se adquieren o modifican habilidades, destrezas, conocimientos, conductas o valores, debido a la experiencia y no a factores madurativos o biológicos, o que dependan exclusivamente del funcionamiento interno del organismo. Prácticamente todas las definiciones de aprendizaje, independiente de las teorías en que se sustenten, tienen en común los siguientes aspectos: 1) se trata de un proceso que conduce a algún resultado, 2) involucra 
cambio o transformación en la persona, ya sea en su comportamiento, en sus estructuras mentales y cerebrales, en sus emociones, etc., 3) se produce como resultado de la experiencia.

Desde la época de los griegos, se ha planteado que el aprendizaje se produce a través de la acción. Según Richards y Rodgers (2001), las actividades se eligen con el propósito de motivar la participación de los estudiantes en el proceso de enseñanza/aprendizaje y son, por lo tanto, la clave para salir del aprendizaje memorístico y fomentar un aprendizaje más significativo. Las acciones y actividades que llevamos a cabo (que transforma el mundo que nos rodea y modifica nuestra experiencia) se expresan de diferentes formas y con distintos niveles de complejidad. Hay actividades que ponen en juego, principalmente, nuestro intelecto, otras nuestra emoción y otras nuestra capacidad física. Hay actividades que se relacionan, simplemente, con el comportamiento que pone de manifiesto una persona a través de sus capacidades; otras, en cambio, proyectan estas capacidades en alguna herramienta, instrumento, artefacto o prótesis, amplificando las posibilidades de transformación que tiene el sujeto en relación al mundo. Actualmente, una cantidad innumerable de actividades se llevan a cabo mediante herramientas digitales que amplifican las capacidades de las personas hasta límites nunca antes conocidos en la historia de la humanidad.

Desde otro punto de vista, las actividades que lleva a cabo un sujeto pueden ser de carácter individual o articularse con las actividades de otros sujetos, formando parte de una acción o trabajo colectivo. Para que exista este trabajo colectivo, se requieren objetivos transpersonales, que den coherencia y cohesión a las acciones individuales. Ya en épocas muy antiguas, diferentes grupos humanos, culturas y civilizaciones han conseguido articular la actividad de las personas para transformar, de acuerdo a sus creencias y aspiraciones, el mundo que les ha tocado vivir (Salas, 2012). Es posible, articular las acciones individuales sobre la base de objetivos colectivos, que son incorporados por cada sujeto en función de una empresa que los trasciende.

Desde el punto de vista del aprendizaje, toda actividad que llevamos a cabo puede constituirse como actividad de aprendizaje, en la medida que responda a objetivos educativos. Una actividad de aprendizaje pone de manifiesto una forma de hacer y, al mismo tiempo, puede estar relacionada con objetivos de aprendizaje, competencias, estándares de desempeño o diferentes nomenclaturas de criterios que se utilicen para determinar lo que se espera que un estudiante aprenda e incorpore. En este contexto, todas las actividades de aprendizaje pueden ser evaluadas y es conveniente que sean evaluadas de manera explícita. Así, la variedad de actividades que pueden programarse y realizarse en el ámbito de la educación, es ilimitada.

Para evaluar las actividades, es necesario observar y describir los resultados que se alcanzan a través de éstas, mediante huellas o evidencias. En el caso de las actividades de aprendizaje en educación, se les llama evidencias de aprendizaje o de desempeño. Las evidencias de desempeño en el ámbito de la educación, como las huellas de cualquier tipo de actividad, son percibidas por alguien que intentará comprenderlas, atribuirles significado y asignarles valor, sobre todo cuando existe un propósito evaluativo. Esto implica que la evaluación de actividades de aprendizaje es, esencialmente, un proceso de comunicación. En un proceso de enseñanza-aprendizaje, por lo tanto, no sólo se requiere que el estudiante "aprenda a hacer", sino también que aprenda a comunicar aquello que ha incorporado como aprendizaje y al mismo tiempo, se necesita que el docente sea capaz de orientar este proceso de comunicación y de recoger, de manera adecuada, la información (evidencias) que el estudiante entrega, cuando sus actividades son valoradas (fig. 1).

\section{HACER COMUNICAR VALORAR}

Actividades
$\left(\begin{array}{c}\text { Significativas } \\ \text { para el } \\ \text { aprendizaje }\end{array}\right)\left(\begin{array}{c}\text { Información } \\ \text { apropiada para } \\ \text { la evaluación }\end{array}\right)\left(\begin{array}{c}\text { Requiere } \\ \text { decodificar y } \\ \text { comprender }\end{array}\right)$

Fig. 1: Hacer, comunicar y valorar

En el ámbito de la educación formal, existen cientos de actividades que se llevan a cabo con el objetivo de fomentar el aprendizaje. Muchas de estas actividades están centradas en la producción de información escrita, que el estudiante entrega mediante diferentes tipos de producto: informes, guías, cuestionarios, apuntes, reportes, producciones literarias, pruebas y exámenes, etc. Es evidente, sin embargo, que no todas las actividades que fomentan el aprendizaje tienen que estar centradas en la producción escrita. También es posible utilizar otros medios para recoger evidencias del trabajo y del aprendizaje de los estudiantes: imágenes de fotografía y de video, registros de audio, presentaciones multimediales, mapas mentales, objetos virtuales como maquetas, planos, figuras tridimensionales, etc. 


\section{Modelo de autoevaluación asistida, basado en el uso de plataformas digitales}

Si, como dijera Ávalos, B. (2011), entendemos la formación docente como un proceso complejo y permanente de modelación de prácticas y del pensamiento, la metodología de enseñanza y de evaluación que se utiliza con los estudiantes no solo repercute en el aprendizaje de determinadas materias, sino, también, ejemplifica una forma de acción y de desempeño del profesor en el aula. Según Linda Darling-Hammond (2015), existe un creciente interés, por parte de educadores y evaluadores en elaborar formas alternativas de evaluación, que permitan atender la complejidad de la enseñanza y que produzcan datos válidos de aptitud pedagógica. Esta idea es reforzada por Agudelo-Torres J, F. Rojas-Restrepo, E. Ocampo-Ruiz, y S. Clavijo-Zapata (2018), quienes plantean que ir más allá de la evaluación como un mero instrumento de verificación, además de ser una necesidad, exhorta a los educadores a "generar nuevas visiones, nuevas metáforas y neófitas prácticas valorativas", para construir una práctica evaluativa acertada, prudente y ética. Ahora bien, cuando la evaluación de aprendizajes recoge los juicios de valor que realizan los estudiantes en torno a sus propios logros y resultados de aprendizaje, nos encontramos frente al concepto de autoevaluación. Incorporar estos juicios de valor en el proceso de evaluación, enriquece, significativamente, la planificación de los procesos de enseñanza-aprendizaje y favorece la autorregulación del estudiante en torno a los objetivos, las estrategias, operaciones mentales y acciones necesarias para dar respuesta a tareas y criterios de evaluación asociados a dichas tareas (Sanmartí, 2007).

Para Heidi Andrade y Ying Du (2007), los conceptos erróneos sobre la autoevaluación del estudiante son comunes. Se suele reducir la "autoevaluación" a la idea de que los estudiantes se califiquen a sí mismos, sin tomar en cuenta que esta práctica también se relaciona con la oportunidad de generar un proceso de reflexión sobre la calidad de propio trabajo para aprender más, hacer mejoras y, quizás, incluso, para obtener una calificación más alta. Stiggins (2001), plantea que la autoevaluación debe estar basada en criterios y estos criterios deben ser tan transparentes, que los estudiantes puedan llegar a evaluar su propio trabajo de la misma manera que su profesor. La mayoría de los investigaciones disponibles, informan que los criterios utilizados en autoevaluación son definidos por un profesor o instructor, sin embargo, algunos autores sostienen que es recomendable que estos criterios sean definidos en conjunto, por el docente y por los estudiantes (Andrade, H y Du, Y, 2007). Otra característica de la autoevaluación, es su capacidad de promover el aprendizaje mediante la retroalimentación y la reflexión de los alumnos (Horner y Shwery, 2002). Así mismo, la autoevaluación implica un monitoreo y un acompañamiento regular de los procesos de pensamiento, que se producen, mediante el desempeño de tareas, a medida que éstas se llevan a cabo (Andrade y Boulay, 2003). Cabe señalar, por último, que los estudios relacionados con la evaluación de aprendizajes, ya sea mediante heteroevaluación, coevaluación o autoevaluación, han estado centrados, principalmente, en el ámbito de la escuela. Aún existe poca información fundamentada en experiencias de educación superior (Manuel J. Acebedo- Afanador, Inmaculada Aznar-Día y Francisco J. Hinojo-Lucena, 2017).

El modelo de autoevaluación asistida se basa en el concepto de evaluación auténtica. Para autores(as) como Darling-Hammond y Snyder (2000), la evaluación auténtica parte de la base que existe un espectro amplio de desempeños que el estudiante puede demostrar, más allá del limitado conocimiento que se puede evidenciar mediante un examen oral o escrito, ya sea de respuesta breve o extensas. Según Condemarín y Medina (2000), la evaluación auténtica tiene sus bases en la concepción de aprendizaje significativo de Alexandron G, M. Armoni, M. Gordon y D. Harel (2017), en la perspectiva cognoscitiva de Novak y Gowin (2002) y en la práctica reflexiva de Schön (1987) y Shin (2006). El modelo de autoevaluación asistida consiste, básicamente, en entregar al estudiante y la posibilidad de evaluar su trabajo y su aprendizaje, contando con el acompañamiento, la asesoría y la supervisión permanente del profesor. No se trata, simplemente, de pedir a los estudiantes que "se pongan una nota" a sí mismos, sino de entregarles un rol protagónico en la valoración de su desempeño, tanto, desde un punto de vista de evaluación formativa como de evaluación sumativa. El docente, en lugar de ser un juez que emite un veredicto en torno al aprendizaje y al logro del estudiante, es visto como un especialista, que además de configurar actividades apropiadas para que el estudiante aprenda, le entrega las herramientas para que pueda evaluar su actividad y su aprendizaje. Así mismo, el docente no es un simple replicador de conocimiento que producen "otros", sino que el mismo es un investigador: aprende de su propia práctica y de la retroalimentación que va obteniendo de sus estudiantes.

Por otra parte, en la actualidad se cuenta con herramientas computacionales que permiten amplificar y mejorar sustantivamente las posibilidades de memoria, de cálculo, de expresión verbal y de comunicación. Todas estas capacidades, existentes a través de la interacción del ser humano con la computadora, pueden constituir un aporte al trabajo que un profesor necesita realizar para retroalimentar, apoyar y valorar las actividades de aprendizaje que llevan a cabo los estudiantes. "Las posibilidades de las herramientas TIC, en cuanto a interactividad y adaptación a las necesidades educativas de los estudiantes, facilitan los procesos de aprendizaje de acuerdo con los distintos estilos predominantes en el alumnado. La interacción, por lo tanto, de nuevas tecnologías y estilos de aprendizaje es un campo de estudio de gran interés, en el ámbito universitario, ya que un mayor logro y rendimiento académico pasa por una individualización de la enseñanza, 
en la que las TIC juegan un papel determinante". (Cózar-Gutiérrez, R. M.V. De Moya-Martínez, J.A. Hernández-Bravo y J.R. Hernández-Bravo, 2016).Por ejemplo, aquel rito de crear pruebas "objetivas" de contenidos, aplicarlas en papel, corregirlas una por una, y utilizar los resultados para calificar a los estudiantes, hoy puede sustituirse por un tipo de ejercicio mucho más eficaz y flexible, sobre todo desde el punto de vista de la retroalimentación: a través del uso de plataformas educativas, el profesor puede crear baterías de preguntas que utiliza para configurar distintas pruebas en diferentes momentos. Estas pruebas pueden autocorregirse y calcular puntuaciones, de manera automática. Los estudiantes tienen la posibilidad de responderlas el número de veces que se estime conveniente.

Así mismo, las plataformas educativas virtuales nos permiten registrar, ordenar y valorizar todo tipo de actividades llevadas a cabo por los estudiantes, contando con herramientas de búsqueda y procesamiento de la información, que pueden ser utilizadas para automatizar el trabajo del docente, sobretodo en relación a aquellas tareas repetitivas que demandan una gran cantidad de tiempo y de energía. Por último, estas mismas plataformas, bien manejadas, entregan la posibilidad de ampliar y diversificar las instancias de comunicación entre las personas que participan de un determinado proceso formativo, generando alternativas que complementan la clase presencial, o, lisa y llanamente, la sustituyen, como en el caso de la educación elearning.

\section{Planificación}

En el modelo de autoevaluación asistida, basado en el uso de plataformas digitales, es necesario llevar a cabo una rigurosa preparación inicial de las actividades de aprendizaje y de los procedimientos y criterios de evaluación, en función de los objetivos o de las competencias definidas para alcanzar en el proceso. La organización del trabajo del curso, se realiza por actividad, no por contenido. Esto, debido a que el proceso debe ordenarse en función del hacer del estudiante. La premisa básica es que "se aprende haciendo". Incluso el saber conceptual se adquiere porque el sujeto, para aprender, algo hace con los conceptos: los relaciona entre sí, los aplica en situaciones concretas, los traduce a diferentes tipos de lenguaje. Ahora bien, para la preparación de un curso bajo el modelo de autoevaluación asistida, se recomienda llevar a cabo los siguientes pasos: distribución de las actividades en unidades temporales, desglose de las actividades en acciones y en criterios cuantitativos de evaluación: una vez que hemos, e Integración de las diferentes formas de evaluación en un curso o módulo.

Distribución de las actividades en unidades temporales: una vez definidas todas las actividades del curso, es necesario distribuirlas en unidades temporales, de acuerdo a los objetivos o competencias considerados en el curso, y determinar cuáles interesa que sean evaluadas y de qué manera. La típica unidad temporal para organizar las actividades es la clase. Como algunas actividades pueden ser abordadas fuera de la clase presencial (tareas) aparecen nuevas unidades temporales, como la semana o el intervalo de días que hay entre una clase y otra.

Desglose de las actividades en acciones y en criterios cuantitativos de evaluación: una vez que hemos definido las actividades, estamos en condiciones de desarrollar los criterios de evaluación y sus respectivos indicadores. Posteriormente, se elabora una escala, que utilizaremos para ponderar las actividades y los criterios e indicadores de evaluación. Finalmente, ponderamos los criterios e indicadores de evaluación, en función de su relevancia para el aprendizaje.

Integración de las diferentes formas de evaluación en un curso o módulo: una vez que han sido preparadas las actividades de aprendizaje y los procedimientos y criterios de evaluación, se procede a configurar estos elementos en alguna plataforma educativa virtual. En el caso del modelo de autoevaluación asistida, que se presenta en este estudio, la plataforma utilizada fue Moodle 3.1.2.Para configurar las actividades en una plataforma educativa, se recomienda seguir el esquema de distribución de actividades en unidades temporales: crear una o varias unidades mayores, incorporar clases dentro estas unidades y, finalmente, incorporar actividades dentro de las clases. En cada unidad temática, se configuran las clases, identificando su nombre, a través de la herramienta etiquetas. Finalmente, bajo cada clase, se incorporan las actividades programadas en la estructura del curso.

\section{Dispositivos para la evaluación}

Los dispositivos de evaluación permiten obtener información en torno al aprendizaje que se adquiere, en un proceso formativo, mediante evidencias, relacionadas con el desempeño o con acciones específicas. Estas evidencias deben permitir la observación de conocimientos, habilidades, destrezas y actitudes que una persona posee. Pueden, básicamente, clasificarse en: evidencias de conocimiento, de proceso o de producto. Los dispositivos de evaluación, que han sido utilizados en el modelo de evaluación asistida, son los siguientes: 
Elaboración preliminar del contrato de aprendizaje: el contrato de aprendizaje o contrato didáctico, no sólo permite aclarar la forma de trabajo y de evaluación que se adoptará en un determinado curso, sino que también favorece un tipo de participación basada en la autonomía y en la responsabilidad del estudiante hacia su propio proceso. En términos generales, se trata de un acuerdo formalizado entre el profesor y el estudiante, donde se hacen explícitos los compromisos de ambas partes, durante un período de tiempo determinado.

Memorias cualitativas del proceso de aprendizaje: para recoger y analizar información en torno al aprendizaje de los estudiantes, necesitaremos implementar determinados mecanismos para construir un registro de observaciones, reflexiones, entrevistas y evidencias de trabajo, relacionadas con el desarrollo de las actividades. En este sentido, se considera la construcción de un portafolio digital, por parte de los alumnos, y de un cuaderno de registro, por parte del docente, como memorias cualitativas del proceso de enseñanzaaprendizaje.

Informe final de calificación: al finalizar el curso o módulo, los estudiantes propondrán una calificación final (autoevaluación), completando un informe, con formato de escala de apreciación o rúbrica, que reunirá todas las actividades desarrolladas en el proceso, con sus respectivos criterios e indicadores y una valoración numérica (evaluación sumativa). Esta propuesta deberá ser coherente con las evidencias de trabajo, existentes tanto en el portafolio, como en la plataforma educativa virtual. El profesor deberá cotejar si hay coherencia entre la autoevaluación cuantitativa y las evidencias entregadas en el portafolio, por cada estudiante. Si hay coherencia, mantendrá la calificación que propone el estudiante. Si no la hay, deberá mostrar, claramente, al alumno la inconsistencia de su propuesta, antes de colocar él una nota final diferente. La idea es que el estudiante participe activamente en la evaluación y en la nota final que obtiene en el curso, a través de un proceso de diálogo con el docente, donde existen márgenes de flexibilidad y negociación, pero donde prima la responsabilidad en el juicio, como principio básico.

Tanto la evaluación cuantitativa como la cualitativa se aplican, permanentemente, durante el proceso (evaluación formativa). Por una parte, el estudiante califica su propia actividad, utilizando una pauta que describe criterios e indicadores de evaluación. Esta pauta es calificada por el estudiante, a través de una escala numérica. A su vez, el profesor retroalimenta estas evaluaciones cuantitativas a través de ejemplos, conversaciones colectivas y observaciones a cada participante, preferiblemente, a través de la plataforma.

\section{Actividades desarrolladas por el estudiante y el docente en el modelo de autoevaluación asistida}

A modo de síntesis, en el modelo de autoevaluación asistida, el proceso de actividades, tanto del docente como de los estudiantes, se desarrolla de la siguiente manera:

Tabla 1: Actividades del docente y del estudiante en el Modelo de Autoevaluación Asistida

\begin{tabular}{|l|l|}
\hline \multicolumn{1}{|c|}{ Actividad } & ¿Quién está a cargo? \\
\hline $\begin{array}{l}\text { Preparación inicial de las actividades de aprendizaje y de los procedimientos y criterios } \\
\text { de evaluación }\end{array}$ & Docente \\
\hline Distribución de las actividades en unidades temporales & Docente \\
\hline Desglose de las actividades en acciones y en criterios cuantitativos de evaluación & Docente \\
\hline Integración de las diferentes formas de evaluación en un curso o módulo & Docente \\
\hline Contrato de aprendizaje & Estudiante-docente \\
\hline Desarrollo de las actividades planificadas en el curso & Estudiante \\
\hline $\begin{array}{l}\text { Desarrollo de memorias cualitativas del proceso de aprendizaje, a través de un portafolio } \\
\text { digital }\end{array}$ & Estudiante \\
\hline $\begin{array}{l}\text { Desarrollo de memorias cualitativas del proceso de aprendizaje, a través de un cuaderno } \\
\text { de registro }\end{array}$ & Docente \\
\hline $\begin{array}{l}\text { Elaboración del Informe final de calificación, incorporando criterios de evaluación para } \\
\text { cada actividad del curso }\end{array}$ & Docente \\
\hline Calificación de actividades realizadas en el curso, a través del informe final de calificación & Estudiante \\
\hline $\begin{array}{l}\text { Verificación de las calificaciones para cada actividad, asignadas por el estudiante, en } \\
\text { función de las evidencias recogidas en el curso }\end{array}$ & Docente \\
\hline
\end{tabular}




\section{METODOLOGÍA}

El objetivo principal del estudio fue describir la valoración de los estudiantes en relación al modelo de autoevaluación asistida, basado en el uso de plataformas digitales, quienes participaron de un curso de pregrado en una carrera de pedagogía, donde fue aplicado este modelo. Se optó por un enfoque cualitativo. El paradigma, de acuerdo a la tipología desarrollada por Lüka (2008), es de tipo "interpretativo", por cuanto más que explicar las relaciones causales por medio de hechos objetivos y análisis estadísticos buscó "comprender la realidad", a través de un proceso interpretativo. Se utilizó teoría fundamentada de diseño emergente, obteniéndose los datos a partir de entrevistas aplicadas a los estudiantes. A través de un muestreo no probabilístico intencional, se entrevistó a 14 estudiantes, de un total de 61, que participaron en el curso "Innovaciones Tecnológicas como Recursos Didácticos", cuya evaluación se llevó a cabo mediante la implementación del modelo de autoevaluación asistida, basada en el uso de plataformas digitales.

El grupo de estudiantes entrevistados estuvo conformado por 9 mujeres (64\%) y 5 hombres (36\%), cuyas edades fluctuaban entre los 20 y los 44 años, con un promedio de edad de 24,6 años. Es importante mencionar que un número significativo de los estudiantes de la Universidad Católica del Maule proviene de sectores socioeconómicos medios y medio bajos y de zonas rurales, ya que la universidad está emplazada en una de las regiones más rurales de Chile. Se aplicó una entrevista semi-estructurada a los 14 estudiantes que participaron del estudio, cuyas preguntas fueron validadas a través de juicio de expertos. Cuatro académicos, doctores en educación, revisaron estas preguntas e hicieron algunas sugerencias, que fueron asumidas en su totalidad por los investigadores.

En cuanto al desarrollo de la investigación, en la fase exploratoria se definió la importancia de implementar nuevas formas de evaluar el aprendizaje de estudiantes universitarios, sobretodo en carreras de pedagogía, debido a las nuevas exigencias en el sistema educativo y se detectó la necesidad de recoger información en torno a la aplicación de un modelo de evaluación, que venía aplicándose y perfeccionándose desde el año 2012. El modelo de autoevaluación asistida fue publicado en 2016 a través de un libro (Rioseco, 2016), que describió en detalle su implementación y expuso su fundamento teórico. En la fase de planificación y de entrada al escenario, se decidió escoger como contexto, un curso de pedagogía de tercer año de la Carrera de Inglés, debido, principalmente, a la facilidad de acceso al objeto de estudio.

En la fase de entrada en el escenario, cabe señalar, que el instrumento fue aplicado a los estudiantes de manera voluntaria y anónima, una vez que finalizó el curso y que ya contaban con sus calificaciones en el sistema de notas de la universidad. Previamente, se les explicó el objetivo de la investigación y se les pidió su consentimiento para analizar sus respuestas y, eventualmente, publicarlas a través de un estudio. Para procesar la información recogida, en primer término, se transcribieron los registros obtenidos a información textual, con el propósito de incorporar documentos primarios en el programa de análisis cualitativo Atlas-ti 7.0, que fue la herramienta utilizada para llevar a cabo el análisis cualitativo. Las unidades de registro o "citas" que se incorporaron para el proceso de codificación, se establecieron de manera inductiva, es decir, sobre la base de unidades mínimas con sentido que fueron emergiendo de los textos. Vale decir, se aplicaron procedimientos asociados al diseño emergente de la teoría fundamentada, que efectuó la codificación abierta. A partir de ésta, emergieron las categorías, que fueron conectadas entre sí. A nivel léxico, se tomó en cuenta la implicancia y connotación en el uso de las palabras. A nivel de la pragmática, se consideraron los actos de habla, juicios, etc. (Hernández, et al., 2010).

\section{RESULTADOS}

A continuación se presentan los resultados, tomando en consideración cinco categorías que surgieron a partir del análisis cualitativo. 1) valoración de las actividades, 2) percepción del aprendizaje, 3) percepción de la evaluación, 4) percepción del nivel de exigencia del curso y de la evaluación, y 5) valoración en torno a la propuesta general del curso

\section{Valoración de las actividades}

En términos generales, las actividades del curso son bien valoradas. Algunos estudiantes se refieren al efecto que estas actividades poseen en relación al aprendizaje, destacando que fomentan la participación, el trabajo autónomo, el compromiso y la motivación, el trabajo colaborativo y la capacidad para regular y organizar la propia el trabajo en sí. En esta línea, un estudiante afirma: "todas las actividades realizadas fueron consecuentes a los conocimientos que se quería que las estudiantes adquirieran. En cuanto a mi aprendizaje en particular las actividades que se realizan diariamente permitieron que se obtuviera un aprendizaje significativo, ya que en primer lugar se debía aprender en el aula y luego llevar a cabo lo aprendido de forma autónoma". 
De igual modo, se menciona que las actividades son consecuentes con los objetivos y se presentan de forma innovadora. Esta valoración positiva de las actividades es fundamental en el marco de la propuesta de este curso, ya que el modelo desarrollado, explícitamente, se basa en las actividades como el eje articulador del proceso de enseñanza y aprendizaje.

\section{Percepción del aprendizaje}

Podemos identificar dos ámbitos en que los estudiantes dicen haber adquirido aprendizajes: el primero está relacionado con el uso de herramientas tecnológicas: aplicaciones vinculadas a Google, como Drive para la producción de documentos, Sites, para la creación de páginas web, Blogspot para la generación de Blogs, uso de planillas de cálculo y procesador de texto, etc. La finalidad principal en el uso de estas herramientas era la producción y el manejo de información digital, en función de preparar a los estudiantes para su futuro desempeño como profesionales de la educación, en tareas académicas y administrativas. Un estudiante entrevistado, señaló lo siguiente: "Influyeron en poder fortalecer los conocimientos que tenían en programas en el pc, además de aprender cómo crear páginas de Internet". El segundo ámbito, se relaciona con habilidades pedagógicas y de comunicación para desempeñar la actividad docente. En este sentido, una estudiante dice lo siguiente: las actividades me permitieron conocer nuevas metodologías de enseñanza y contribuyeron para desarrollar mi habilidad para regular y organizar mi trabajo.

\section{Percepción de la evaluación}

En general, la percepción que existe en torno a la evaluación que se llevó a cabo en el curso es positiva. Hay dos opiniones, sin embargo, que consideran que es poco clara y que las calificaciones definitivas no se conocen hasta que finaliza el proceso. Estas opiniones dan cuenta de un hecho real. Hay que considerar que la actual propuesta confronta, de manera premeditada, con el sistema tradicional, basado en la aplicación de exámenes y en la obtención de una calificación final, en función del promedio o la ponderación de los exámenes parciales. No contar con calificaciones parciales estáticas, debido a que los productos que se derivan de las actividades pueden ser mejorados y vueltos a evaluar a lo largo del proceso, genera un cierto nivel de incertidumbre en algunos estudiantes. La gran mayoría de los estudiantes, sin embargo, valora positivamente este sistema de evaluación. Entre otras cosas, destacan que es una forma innovadora: sistemática y diferente de evaluar, que refleja lo que se aprende, dando espacio a la argumentación y disminuyendo el estrés. Por ejemplo, una estudiante afirma: "la evaluación se lleva a cabo de manera innovadora ya que puedo seguir yo misma mi proceso para así mejorar y también ver la evaluación del profesor".

Una evaluación que está orientada a la elaboración de productos que favorece el aprendizaje de programas computacionales, al tiempo que incentiva el compromiso del estudiante y su sinceridad a la hora de autoevaluarse.

\section{Percepción del nivel de exigencia del curso y de la evaluación}

La opinión que existe de los estudiantes en relación al nivel de exigencia del curso, tiende a considerar que es adecuado o alto, coherente con los objetivos, las actividades y los contenidos del curso y ajustado a las características del grupo de estudiantes. Un estudiante, afirma: el nivel de exigencia está acorde a los conocimientos entregados en el curso, además el profesor se encarga de realizar procedimientos para que nosotras como estudiantes podamos adquirir las habilidades necesarias del curso.

Una de las alumnas del curso, sin embargo, piensa que el nivel de exigencia es bajo, por tratarse de una carrera que debe formar profesionales competentes en el sistema escolar.

\section{Valoración en torno a la propuesta general del curso}

En relación a la propuesta general del curso, se destaca que trabaja con programas relevantes para el futuro profesional de los y las estudiantes y promueve la incorporación de diversos conocimientos. Por la forma en que está planteado, podría ser un curso en línea. Como aspectos positivos, se menciona que es un curso enriquecedor, que incorpora contenidos, conocimientos y actividades pertinentes para el rol del educador. La propuesta de enseñanza del curso, se basa en la programación de procedimientos que los alumnos deben aprender y en la incorporación de contenidos que se comprender a través de la práctica. Como aspectos negativos, algunos(as) estudiantes opinan que se imparte muy tarde dentro de la malla de la carrera y que el número de trabajos es excesivo. Finalmente, como recomendación y, en coherencia, con los aspectos negativos, se sugiere impartir este curso en primer año de la carrera y entregar calificaciones a lo largo del semestre. 


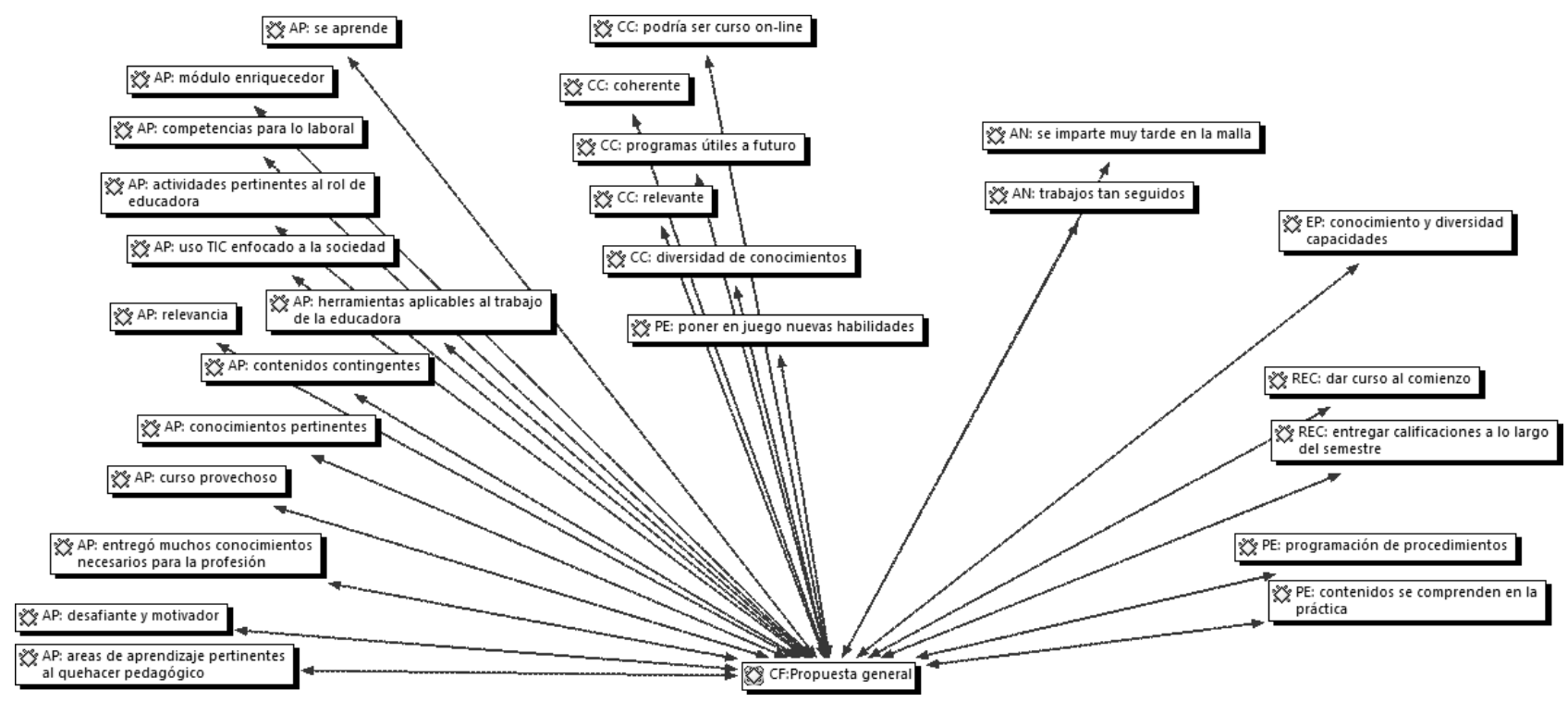

Fig. 2: Valoración de la Propuesta General del Curso

\section{DISCUSIÓN, ANÁLISIS Y REFLEXIONES}

En la línea del estudio de Brown, Andrade y Chen (2015), "Accuracy in student self-assessment: Directions and cautions for research", compartimos la idea de que es necesario crear las condiciones óptimas para maximizar la precisión en los procesos de autoevaluación. Necesitamos tener una mejor comprensión de la naturaleza de la autoevaluación y mejorar la fiabilidad y la confianza que existe en torno a esta modalidad evaluativa. El modelo de autoevaluación asistida, comparte los principios fundamentales de la evaluación auténtica. En términos generales, podemos decir que el modelo de autoevaluación asistida consiste en entregar al estudiante la posibilidad de evaluar su trabajo y su aprendizaje, contando con el acompañamiento, la asesoría y la supervisión permanente del profesor. Al mismo tiempo, incorpora herramientas cuantitativas, propias de la medición, desde una óptica diferente a la estandarización educativa; una óptica que utiliza la cuantificación numérica para asignar valor al desarrollo de las actividades, desde el punto de vista del estudiante, con el fin de promover el aprendizaje. La principal herramienta cuantitativa que se utiliza en este modelo es la pauta de calificación, que identifica criterios de evaluación e indicadores, a los cuales el estudiante asigna un valor numérico, a través de una escala.

De acuerdo a la investigación "Peer / Self Assessment and Student Learning", de Abdou Ndoye (2017), la retroalimentación, la colaboración, y el ambiente de trabajo, facilitado por la autoevaluación, constituyen mecanismos que contribuyen al aprendizaje de los y las estudiantes y promueven la responsabilidad hacia la propia evaluación, entregando elementos que favorecen una mayor conciencia de los requisitos de un curso. En este sentido, es importante, para la proyección del modelo, que los estudiantes investigados hayan valorado positivamente la metodología de evaluación utilizada. Así mismo, la valoración que llevaron a cabo de las actividades desarrolladas, permite inferir que estas actividades cumplieron el objetivo de constituirse como medios a través de los cuales los estudiantes adquirieron aprendizajes, en esferas tanto cognitivas, afectivas, como de conducta o comportamiento (Cooper, 2005).

El uso de herramientas tecnológicas, que los estudiantes investigados declaran haber aprendido a utilizar en este curso, constituye un saber fundamental, que habrá de incidir en su desempeño futuro como docentes, principalmente en lo que se refiere a la capacidad de potenciar diferentes estilos de enseñanza y de aprendizaje, facilitando la interactividad y el acceso flexible a la información. Una implicación importante en el presente trabajo, es la necesidad de los educadores de generar ambientes propicios para el aprendizaje, que fomenten la confianza entre los pares, hacia el docente y hacia el proceso en sí mismo. De esta manera la autoevaluación puede convertirse en una práctica evaluativa genuina, que se lleva a cabo con el propósito de mejorar y no solamente de alcanzar calificaciones altas. Por otra parte, se deben desarrollar criterios claros para promover una reflexión profunda y relevante, en torno al propio desempeño. Los beneficios de la autoevaluación pueden ser maximizados, si las actividades están diseñadas de tal forma que permiten a los estudiantes aplicar sus habilidades evaluativas. En este sentido, se destaca la importancia de dotar de nuevo significado la evaluación educativa, diseñándola para fomentar una mirada responsable del estudiante en torno a su propio desempeño en las actividades que desarrolla, en los productos que genera y en los aprendizajes que adquiere. 
Finalmente cabe señalar, como limitación de este trabajo, que, si bien los y las estudiantes valoraron positivamente la aplicación del modelo de autoevaluación asistida, no todos lograron ver su interconexión con un aprendizaje significativo y de calidad.

\section{CONCLUSIONES}

De los resultados mostrados, de su análisis y de su discusión, se pueden obtener las siguientes conclusiones, en torno a la Aplicación del Modelo de Autoevaluación Asistida Basado en el uso de Plataformas Digitales, en un Curso de Pedagogía: 1) el modelo de autoevaluación asistida, comparte los principios fundamentales de la evaluación auténtica, está basado en el uso de plataformas digitales y busca atender la complejidad en el proceso de evaluación, aprovechando las posibilidades que entregan hoy las TIC; 2) el modelo propuesto, a pesar de que es fácil de implementar, conlleva un cambio en la forma clásica de evaluar, ya que modifica la manera en que se asigna valor a las actividades y al aprendizaje, en sí mismo; 3) en términos generales, los estudiantes valoran positivamente la aplicación del modelo de autoevaluación asistida, destacando la relevancia de las actividades y la forma en que se fomenta el aprendizaje.

\section{REFERENCIAS}

Acebedo- Afanador, M., I. Aznar-Díaz y F. Hinojo-Lucena, Instrumentos para la Evaluación del Aprendizaje Basado en Competencias: Estudio de caso, doi.org/10.4067/S0718-07642017000300012, Inf.Tecnol., 28(3), 107-118 (2017)

Agudelo-Torres, J., F. Rojas-Restrepo, E. Ocampo-Ruiz y S. Clavijo-Zapata, Sobre la Evaluación Escolar y su Ética, doi.org/10.4067/S0718-07642018000500071, Inf. Tecnol., 29(5), 71-80 (2018)

Andrade, H. e Y. Du, Student Responses to Criteria Referenced Self-assessment, doi.org/10.1080/02602930600801928, Educational Administration \& Policy Studies Faculty Scholarship, 4(1), 1-24 (2007)

Andrade, H. y B. Boulay, Gender and the Role of Rubric-referenced Self-assessment in Learning to Write, Journal of Educational Research, 97(1), 21-34 (2003)

Alexandron, G., M. Armoni, M. Gordon y D. Harel, Teaching Scenario-Based Programming: An Additional Paradigm for the High School Computer Science Curriculum, Part 1, Computing in Science \& Engineering, 19 (58) (2017)

Álvarez, E., La cuestión del Sujeto en la Fenomenología de Husserl, ISSN: 1885-1088, Revista Investigaciones Fenomenológicas, Universidad Autónoma de Madrid, 8, 97-149 (2011)

Avalos, B., Teacher professional development in Teaching and Teacher Education over Ten Years, ISSN: 0742-051, Teaching and Teacher Education, 27, 10-20 (2011)

Barrenechea, I., What about Elementary Level Teachers: A Closer Look at the Intersection between Standardization and Multilingualism, Global Education Review, 5(2), 203-222 (2018)

Brown, G., H. Andrade y F. Chen, Accuracy in Student Self-assessment: Directions and Cautions for Research, doi.org/10.1080/0969594X.2014.996523, Assessment in Education Principles Policy and Practice, 22,444-457 (2015)

Condemarín, M. y S. Medina, La Evaluación Auténtica de los Aprendizajes, Andrés Bello, Santiago de Chile (2000)

Cooper, J., Estrategias de Enseñanza, Guía para una Mejor Instrucción, ISSN: 1316-0648, Informe de Investigaciones Educativas, 19, 202-204 (2005)

Cózar-Gutiérrez, R., M.V. De Moya-Martínez, J.A. Hernández-Bravo y J.R. Hernández-Bravo, Conocimiento y Uso de las Tecnologías de la Información y las Comunicaciones (TIC) según el Estilo de Aprendizaje de los Futuros Maestros, doi: 10.4067/S0718-50062016000600010, Formación Universitaria, 9(6), 105-118 (2016)

Darling-Hammond, L. y J. Snyder, Meaningful Learning in a New Paradigm for Educational Accountability: An Introduction, doi.org/10.14507/epaa. v23.1982, Education Policy Analysis Archives, 23(7), 1-9 (2015)

Darling-Hammond, L. y J. Snyder, Authentic Assessment of Teaching in Context, ISSN-0742-051X, Teaching and Teacher Education, 16(5-6), 523-545 (2000)

Hernández, R., C. Fernández y M. Baptista, Metodología de la Investigación, 5ª Ed., McGraw-Hill, México D.F., (2010)

Horner, S. y C. Shwery, Becoming an Engaged, Self-regulated Reader, Theory into Practice, 41(2), 102-109 (2002)

Linn, R.L., Educational Assessment: Expanded Expectations and Challenges, doi.org/10.2307/1164248, Educational Evaluation and Policy Analysis, 15(1), 3 - 38 (1993)

Lipman, P., Más allá de la Rendición de Cuentas: Escuelas para Nuevas Formas de Vida, ISSN 0718-4212, Revista Docencia, 38, 26-43 (2009)

Lüka, I., Development of students' ESP competence and educators preferred activity in tertiary level tourism studies: In I.Zogla (Ed.), Association for teacher education in Europe spring university conference teacher of the $21^{\text {st }}$ century, Quality education for quality teaching university of Latvia, 689-987, Riga, Latvia, (2008)

Maturana, H., Emociones y Lenguaje en Educación y Política, Ed. Dolmen Ensayo, Santiago de Chile (2001) 
Mojarro, A., D. Rodrigo, M. Etchegaray y Centeno, Educación Personalizada través de E-Learning, ISSN: 1390-325X, Alteridad, Revista de Educación, 10(1), 21-30 (2015)

Ndoye, A., Peer / Self Assessment and Student Learning, ISSN: 1812-9129, International Journal of Teaching and Learning in Higher Education, 29(2), 255-269 (2017)

Novak, J. y D. Gowin, Learning how to Learn, 9-20, Cambridge University Press, USA (2002)

Quaas, C. y N. Crespo, ¿Inciden los Métodos de Enseñanza del Profesor en el Desarrollo del Conocimiento Metacomprensivo de sus Alumnos?, doi.org/10.4067/S0718-09342003005400007, Revista Signos, 36(54), 225-234 (2003)

Richards, J. y T. Rodgers, Approaches and Methods in Language Teaching. A Description and Analysis, Cambridge University Press, Cambridge (2001)

Rioseco, M., De la Domesticación hacia una Evaluación Libertaria, Virtual Ediciones, Chile (2016)

Robbins, P.S. y A. T. Judge, Essentials of Organizational Behavior, 2nd Ed., 55. Pearson, South Africa (2009)

Salas, J., Historia General de la Educación, Red Tercer Milenio, México (2012)

Sanmartí, N., 10 Ideas Clave, Evaluar para Aprender, Graó, Barcelona (2007)

Schön, D., Educating the Reflective Practitioner, 355, San Francisco, Jossey-Bass Publishers, CA, (1987)

Shin, J.S., Learning to Teach Writing through Tutoring and Journal Writing, doi.org/10.1080/13450600500467621, Teachers and Teaching, Theory and Practice, 12(3), 325-345 (2006)

Stiggins, R. y J. Chappuis, Student-involved Classroom Assessment to Close Achievement Gaps, Theory into Practice, 44(1), 11-18 (2001) 
